murine colonization testing for immune modulatory properties of individual strains.

References:

[1] Azzouz et al. Ann Rheum Dis 2019 78(7):947-56

Disclosure of Interests: : Gregg Silverman Consultant of: Work with industry is unrelated to the topic in this abstract, Doua Azzouz: None declared, Ze Chen: None declared, Jing Deng: None declared, Zhi Li: None declared, David Fenyo: None declared, Alexander Alekseyenko: None declared

DOI: 10.1136/annrheumdis-2020-eular.5091

\section{THU0240 SELECTIVE DEPLETION OF AUTOREACTIVE B CELLS USING A PEPTIDE VACCINATION APPROACH}

R. Singh ${ }^{1} .{ }^{1}$ UCLA, Autoimmunity and Tolerance Laboratory, Department of Medicine / Rheumatology, Los Angeles, United States of America

Background: B cell depletion is currently approved or being tested in clinical trials for the treatment of many autoimmune diseases including rheumatoid arthritis, myositis, systemic sclerosis, and lupus. We previously demonstrated that the heavy chain variable regions $\left(\mathrm{V}_{\mathrm{H}}\right)$ of anti-DNA antibodies contain epitopes that can bind $\mathrm{MHC}$ class I molecules. ${ }^{1}$ Vaccination of autoimmune-prone NZB/NZWF1 mice with plasmid DNA vectors carrying minigenes that encode such epitopes induced $C D 8^{+}$cytotoxic $T$ lymphocytes (CTL) that killed anti-DNA antibody-producing $B$ cells, reduced serum antiDNA antibody levels, retarded the development of nephritis, and improved survival. ${ }^{1}$ Treatment with peptides alone did not induce such $\mathrm{CD} 8^{+} \mathrm{T}$-cells, as we and others have reported impaired CD8+ regulatory and CTL responses in mice and humans. ${ }^{2,3}$ Here, we asked if we could overcome such impairment using synthetic oligodeoxynuleotides containing unmethylated cytidine-phosphate-guanosine dinucletides (CpG-ODN) that can enhance innate and adaptive immunity.

Objectives: To determine if peptides representing MHC class I binding epitopes in the autoantibody $V_{H}$ regions conjugated to $\mathrm{CpG-ODN}$ will elicit $\mathrm{CD} 8^{+} \mathrm{CTLs}$ that will ablate autoantibody-producing B cells, reduce serum autoantibody levels, and retard the development of autoimmune disease.

Methods: We first sceeened $V_{H}$ regions of anti-dsDNA and other autoantibodies for MHC class I-binding epitopes uisng various bioinformatic approaches, and then verified the binding using cellular binding approaches. We immunized lupus-prone (NZB/NZW F1) mice that develop lupus that mimics human lupus with CpG-ODN or a control ODN, and assessed CTL responses. We then treated these mice with CpG-ODN conjugated to MHC class I-binding $\mathrm{VH}$ epitopes. We monitored lupus mice for proteinuria, serum anti-dsDNA antibodies, and survival.

Results: Immunization with CpG-ODN corrected the impairment in peptide-specific CTL responses in lupus-prone mice. The CpG-ODN conjugated with MHC class I-binding, anti-dsDNA antibody $\mathrm{V}_{\mathrm{H}}$-derived epitopes induced potent peptide-specific CTL responses against autoreactive $B$ cells that expressed the respectie epitopes, and against $B$ cells from diseased lupus-prone mice. The animals treated with CpG-ODN-peotde conjugates had reduced serum antiDNA antibody levels and proteinuria, significantly delayed development of nephritis, and improved survival as compared to animals injected with a control ODN.

Conclusion: Seletive ablation of autoreactive B cells by CTLs induced by peptide vaccines conjugated to CpG-ODN represents a novel approach to treat autoantibody-mediated diseases

References:

[1] Fan G and Singh RR. J Exp Med 2002;196:731-741.

[2] Singh RR. Curr Opin Rheumatol 2000;12:399-406.

[3] Singh RR. Mol Immunol 2004;40:1137-1145.

Disclosure of Interests: : None declared

DOI: 10.1136/annrheumdis-2020-eular.2678

\section{THU0241 ALTERATIONS OF THE FECAL MICROBIOTA ASSOCIATED WITH EXACERBATION OF LUPUS ACTIVITY BY HCMV PP65 422-439 IMMUNIZATION IN NZB/W F1 MICE}

Y. C. Tsai ${ }^{1}$, A. H. Hsieh ${ }^{1}$, L. C. Wang ${ }^{1}$, I. J. Chou ${ }^{2,3}$, W. Y. Tseng ${ }^{4,5}$, K. H. Yu' ${ }^{1}$ S. F. Luo ${ }^{1}$, C. F. Kuo ${ }^{1,6} .{ }^{1}$ Chang Gung Memorial Hospital, Division of Rheumatology, Allergy and Immunology, Taoyuan, Taiwan, Republic of China: ${ }^{2}$ Chang Gung Memorial Hospital, Division of Paediatric Neurology, Taoyuan, Taiwan, Republic of China; ${ }^{3}$ University of Nottingham, Division of Clinical Neurology, School of Medicine, Nottingham, United Kingdom; ${ }^{4}$ University of Oxford, Kennedy Institute, Oxford, United Kingdom; ${ }^{5}$ Chang Gung Memorial Hospital, Division of Rheumatology, Allergy and Immunology, Keelung, Taiwan, Republic of China; ${ }^{6}$ Chang Gung Memorial Hospital, Center for Artificial Intelligence in Medicine, Taoyuan, Taiwan, Republic of China
Background: Systemic lupus erythematosus (SLE) is a multifactorial disease. Gut microbiota is an important environmental factor for $\mathrm{SLE}^{1}$. The perturbation of gut microbiota is often observed at onset or during the disease course. The fragment of HCMV phosphoprotein $65_{422-439}\left(\mathrm{HCMVpp65}{ }_{422-439}\right)$ containing B cell epitopes has been reported to elicit humoral immunity and accelerate the autoimmune response in murine lupus ${ }^{2,3}$. However, little is there to know about the interplay between viral trigger for SLE and the change of gut microbiota during lupus progression.

Objectives: By using a murine lupus model with NZB/W F1, we investigated the differential alteration in gut microbiota associated with the progression of lupus disease in HCMVpp65

Methods: Ten weeks-old NZB/W F1 mice were given or not given an intraperitoneal injection of $100-\mu \mathrm{g} \mathrm{HCMVpp65} 5_{422-439}$ peptide biweekly for four times. Fecal samples, urine and blood of mice were collected once every two weeks followed by 16S rRNA genes sequencing and ELISA tests. The pathological investigation of renal tissue from sacrificed mice was conducted at 24 weeks of mice age. Statistical analysis for dynamics and alteration of the gut microbiota as well as functional prediction of bacterial communities related to the progression of lupus-like activity was performed. Results: HCMVpp65 ${ }_{422-439}$ immunization results in the onset of lupus-like activities in NZB/W F1 mice with a higher titer of anti-dsDNA antibody, creatinine and proteinuria, and severe glomerular damage (figure 1). Also, higher diversity and increased family abundance of several bacterial species were observed in HCMVpp65 ${ }_{422-439}$ immunized mice (Table 1 and Figure 2a). The predicted metagenomic taxonomic profile in NZB/W F1 mice showed statistically significant enrichment of flagellar assembly, bacterial motility, and chemotaxis (Figure $2 b)$. Spearman's correlation analysis revealed that a significant association between the increased relative family abundance for Saccharimonadaceae, Marinifilaceae, Desulfovibrionaceae, and Rikenellaceae and HCMVpp65 ${ }_{422-439}$ induced lupus-like activity in NZB/W F1 mice (Figure 2c).

Table 1. Significant test of microbial community structure between two groups

\begin{tabular}{|c|c|c|c|c|c|c|}
\hline \multirow[b]{2}{*}{ Groups } & \multicolumn{2}{|c|}{ MRPP } & \multicolumn{2}{|c|}{ Adonis } & \multicolumn{2}{|c|}{ Anosim } \\
\hline & $E-\Delta$ & $\mathrm{P}$ & $\mathrm{R}^{2}$ & $\mathrm{P}$ & $\mathrm{R}$ & $P$ \\
\hline Pre-disease vs. Control & 0.43 & 0.1 & 0.77 & 0.1 & 1 & 0.112 \\
\hline Pre-disease vs. HCMVpp65 & 0.52 & 0.007 & 0.55 & 0.009 & 0.97 & 0.01 \\
\hline Control vs. HCMVpp65 & 0.45 & 0.013 & 0.35 & 0.014 & 0.58 & 0.019 \\
\hline
\end{tabular}

Conclusion: Our results demonstrated that HCMVpp65 $5_{422-439}$ immunization induced the change in gut microbiota composition and suggested the association of gut microbiota alteration with lupus-like activity in NZB/W F1 mice.

References:

[1] Azzouz D, Omarbekova A, Heguy A, Schwudke D, Gisch N, Rovin BH, et al. Lupus nephritis is linked to disease-activity associated expansions and immunity to a gut commensal. Ann Rheum Dis. 2019;78(7):947-56.

[2] Sebastiani GD, Iuliano A, Canofari C, and Bracci M. Cytomegalovirus infection in Systemic Lupus Erythematosus: report of four cases challenging the management of the disease, and literature review. Lupus. 2019;28(3):432-7.

[3] HoHsieh A, Wang CM, Wu YJ, Chen A, Chang MI, and Chen JY. B cell epitope of human cytomegalovirus phosphoprotein 65 (HCMV pp65) induced antidsDNA antibody in BALB/c mice. Arthritis Res Ther. 2017;19(1):65

Figure
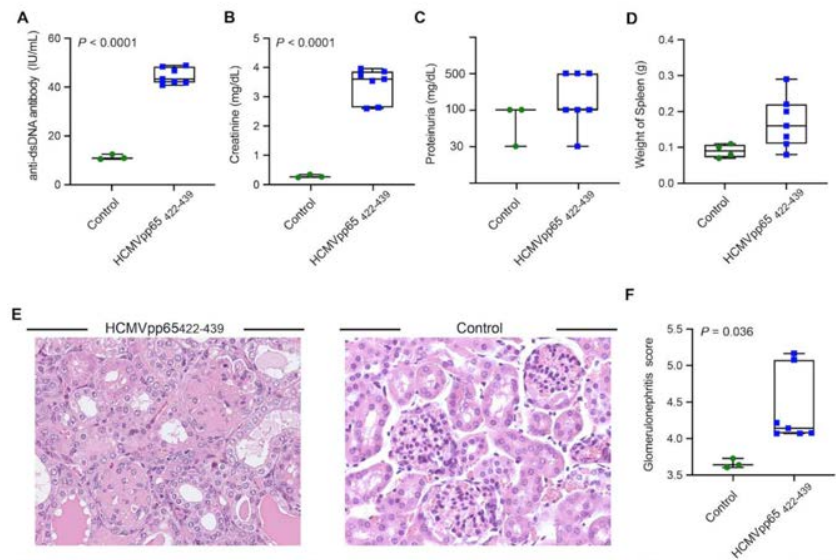

HCMVpp65422-439 immunization accelerates the SLE disease progression in NZBWW F1 mice. ELISA analysis for the levels of (A) representative photograph and diagram shows the size of spleen collected from both groups. (E) Hematoxylin and eosin staining control and immunized mice. 